\title{
PLAU directs conversion of fibroblasts to inflammatory cancer-associated fibroblasts, promoting esophageal squamous cell carcinoma progression via uPAR/Akt/NF-kB/LL8 pathway
}

\author{
Lingling Fang ${ }^{1}$, Yun Che ${ }^{1}$, Chaoqi Zhang ${ }^{1}$, Jianbing Huang ${ }^{1}$, Yuanyuan Lei', Zhiliang Lu', Nan Sun ${ }^{1}$ and Jie He $\mathbb{B}^{1}$
}

\begin{abstract}
Cancer-associated fibroblasts (CAFs) plays an important role in the tumor microenvironment. The heterogeneity of CAFs affects the effect of CAFs on promoting or inhibiting tumors, which can be regulated by other cells in the tumor microenvironment through paracrine methods. The urokinase-type plasminogen activator (PLAU) system mediates cell proliferation, migration, adhesion, and other functions through the proteolytic system, intracellular signal transduction, and chemokine activation. PLAU promotes tumor progression in many tumors. We explored the function of PLAU in ESCC and the influence of PLAU secreted by tumor cells on the heterogeneity of CAFs. We found that PLAU is highly expressed in ESCC, which is related to poor prognosis and can be used as a prognostic marker for ESCC. Through lossof function and gain-of function experiments, we found that PLAU promoted ESCC proliferation and clone formation via MAPK pathway, and promotes migration by upregulating Slug and MMP9, which can be reversed by the MEK 1/2 inhibitor U0126. At the same time, through sequencing, cytokine detection, and RT-qPCR verification, we found that tumor cells secreted PLAU promoted the conversion of fibroblasts to inflammatory CAFs, which upregulated expression and secretion of IL8 via the UPAR/Akt/NF-KB pathway. The IL8 secreted by CAFs in turn promotes the high expression of PLAU in tumor cells and further promoted the progression of ESCC. In summary, PLAU was not only a prognostic marker of ESCC, which promoted tumor cell proliferation and migration, but also promoted the formation of inflammatory CAFs by the PLAU secreted by tumor cells.
\end{abstract}

\section{Introduction}

Esophageal cancer is one of the most common cancers in the world ${ }^{1}$. In 2015, there were an estimated 478,000 new cases and 375,000 deaths in China ${ }^{2}$. Esophageal cancer is highly malignant, and the prognosis is poor. The five-year survival rate is $15-25 \%^{3}$. Esophageal squamous cell carcinoma (ESCC) is the main pathological type of esophageal cancer. Surgery combined with radiotherapy

Correspondence: Nan Sun (sunnan@vip.126.com) or

Jie He (prof.jiehe@gmail.com)

'Department of Thoracic Surgery, National Cancer Center/National Clinical Research Center for Cancer/Cancer Hospital, Chinese Academy of Medical Sciences and Peking Union Medical College, Beijing, China

Edited by Ivano Amelio and chemotherapy is the main treatment for ESCC. However, drug resistance, recurrence, and metastasis are prone to appear after treatment, and the overall quality of life and prognosis of patients are poor.

Cancer-associated fibroblasts (CAFs) are an important component of the tumor microenvironment ${ }^{4}$. They mainly act by secreting cytokines, changing the extracellular matrix, and interacting with tumor cells and other cells $^{4-6}$. Many studies have confirmed that CAFs play an important role in the occurrence, development, drug resistance, and metastasis of ESCC by secreting various cytokines, including HGF, IL-6, CXCL1, and PAI-1 ${ }^{7-10}$. There is enough evidence to prove that CAFs play an important role in tumor progression and may represent

\section{(c) The Author(s) 2021}

(c) (i) Open Access This article is licensed under a Creative Commons Attribution 4.0 International License, which permits use, sharing, adaptation, distribution and reproduction cc) in any medium or format, as long as you give appropriate credit to the original author(s) and the source, provide a link to the Creative Commons license, and indicate if changes were made. The images or other third party material in this article are included in the article's Creative Commons license, unless indicated otherwise in a credit line to the material. If material is not included in the article's Creative Commons license and your intended use is not permitted by statutory regulation or exceeds the permitted use, you will need to obtain permission directly from the copyright holder. To view a copy of this license, visit http://creativecommons.org/licenses/by/4.0/. 
new targets for tumor therapy ${ }^{11}$. Targeting CAFs not only inhibits the growth of tumor "seeds" but also modifies the "soil" to generate a microenvironment that inhibits tumor proliferation and metastasis ${ }^{4}$. Although it is generally believed that CAFs mainly play a role in promoting tumors, some studies have found that the targeted elimination of CAFs in pancreatic cancer leads to tumor deterioration and progression. This finding demonstrates that the greatest challenge of targeting CAFs to treat tumors is the heterogeneity of CAFs ${ }^{11-15}$. In addition, as prognostic markers, CAFs have also demonstrated the opposite results in many studies ${ }^{11,13}$. CAFs can be divided into different subgroups, such as tumor-promoting CAFs (pCAFs), tumor-inhibiting CAFs (rCAFs), neutral CAFs (nCAFs), inflammatory CAFs (iCAFs), and myofibroblasts (myCAFs) ${ }^{16-18}$.

The urokinase-type plasminogen activator (PLAU) system includes PLAU, urokinase plasminogen activator receptor (UPAR) and its inhibitor PAI-1. This system mediates cell proliferation, migration, adhesion, and other functions through the proteolytic system, intracellular signal transduction, and chemokine activation. This system is related to a variety of pathological and physiological processes and plays an important role in tumor occurrence and development ${ }^{19}$. PLAU is overexpressed in many tumors and plays an important role in tumor development and metastasis ${ }^{20-22}$. PLAU is a biomarker and prognostic indicator of breast cancer, which promotes trastuzumab resistance ${ }^{23}$. In addition, exosomes overexpressing of mir-23b and miR-320b in epithelial cells in breast cancer upregulate the expression of PLAU ${ }^{24}$. Our previous studies have confirmed that CAF-derived PAI-1 promotes ESCC cell proliferation and clone formation, and inhibits ESCC cell apoptosis caused by cisplatin. In vivo, PAI-1 can promote ESCC cell subcutaneous tumor formation in nude mice and cisplatin resistance. PAI-1 expression in the drug-resistant CAFs group was significantly increased and associated with poor progressionfree survival $(\mathrm{PFS})^{8}$. This study aims to explore the function of PLAU in ESCC tumor cells and the influence of PLAU secreted by tumor cells on CAF heterogeneity in the microenvironment.

\section{Materials and methods}

\section{Patients and tumor samples}

A paraffin-embedded ESCC microarray contained 55 ESCC tissue with 50 adjacent tissues was purchased from Outdo Biotech (HEsoS105Su01) for immunohistochemistry studies. Ten fresh ESCC tumor and paratumor tissues were obtained from our hospital for isolation of CAFs in 2019. All patients signed informed consent. Our study was approved by the Committee for the Ethics Review of Research Involving Human Subjects of the
Cancer Hospital of the Chinese Academy of Medical Sciences.

\section{Immunohistochemistry (IHC) and scoring}

In brief, IHC was performed as reported in a previous study. Anti-PLAU antibody (diluted 1:100, ab169754, Abcam) was used. The final immunoreactivity score (IRS) was the product of staining intensity and percentage of positive cells.

\section{Cell culture and stable cell construction}

As previously described, the ESCC cell lines KYSE-30, KYSE-70, KYSE-140, KYSE-150, KYSE-180, KYSE-450, and KYSE-510 were cultured in RPMI 1640 medium

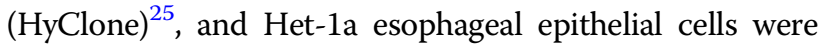
cultured in BEGM BulletKit medium (Lonza/Clonetics). MRC-5 (Medical Research Council cell strain-5, KG508, Keygene) was cultured in MEM (minimum Eagle's medium) with $10 \%$ fetal bovine serum, $1 \%$ nonessential amino acid and $1 \mathrm{mM}$ sodium pyruvate. Full-length PLAU cDNA was synthesized by SyngenTech (China) and ligated into a pLV-CMV-Puro vector. Two short hairpin RNA (shRNA) oligonucleotides (5'-GCATGACTTTGACTGGAATTG-3' and 5'-GCAGTAGAGTCATCTCCATCA-3') were inserted into pLV-Puro (SyngenTech, China). The negative control (NC) sequence was 5'-AAACGTGACACGTTCG GAGAA-3'. As previously described, we constructed ESCC cells with knockdown or overexpression of $P L A U$ and controls. PLAU expression in infected cells was confirmed by RT-qPCR and western blot $96 \mathrm{~h}$ after infection.

\section{Isolation CAFs and normal fibroblasts (NFs)}

Homogeneous CAFs or NFs were isolated from fresh tumor or paratumor tissues and were identified using the cellular immunofluorescence marker $\alpha$ SMA as noted in our previous study ${ }^{8}$. The patients' characteristics was in Supplementary Table 2. All CAFs and NFs used in the study were grown for no more than ten passages.

\section{Collection of conditional medium (CM)}

In brief, the CM was collected after shPLAU-1, shPLAU-2, -vec KYSE-30, and KYSE-450 cells and PLAU, vector KYSE-180 and KYSE-450 cells were cultured in serum-free medium for $24 \mathrm{~h}$. The CM of CAFs was collected as follows. After exposure to various treatments (stimulated by drugs or CM of tumor cells, or cocultured with tumor cells), the medium was replaced with the serum-free medium, and cells were incubated for $24 \mathrm{~h}$. $\mathrm{CM}$ was centrifuged at $1000 \times g$ for $5 \mathrm{~min}$ for further experiments. For western blot, CM was concentrated 40fold using a Centricon Centrifugal filter (Millipore, USA). For cell stimulation, CM was sterile filtered and diluted once with the medium. 


\section{Drugs}

Briefly, $10 \mu \mathrm{M}$ U0126 (catalog no. S1102, Selleck) was added to the cell culture for $1 \mathrm{~h}$ to inhibit MEK1/2 in vitro. CAFs were treated with $2 \mathrm{ng} / \mathrm{ml}$ recombinant PLAU (catalog no. ab167764, Abcam) for $24 \mathrm{~h}$. Briefly, $10 \mu \mathrm{M}$ IPR-803 (catalog no. HY-111192, Selleck) was added $1 \mathrm{~h}$ prior to inhibiting PLAU-uPAR and before any other treatments. Recombinant IL8 (catalog no. 500-P28, Peprotech) was used to at final concentrations of $5 \mathrm{ng} / \mathrm{ml}$ unless specified. Times of treatments were $24 \mathrm{~h}$ unless specified.

\section{Coculture system}

For the coculture system, WT KYSE-30 and KYSE-450 cells were seeded in a 24-well plate, and CAFs with recombinant PLAU and IPR-803 treatments were placed in the upper chamber with Matrigel with a $0.4-\mu \mathrm{m}$ pore size (catalog no. 3412, Corning). Cells were cocultured for $48 \mathrm{~h}$. Then, KYSE-30 and KYSE-450 cell proliferation was detected using the CCK8 assay. In another experiment, CAFs treated with IPR-803 or PBS were seeded in a 24well plate, and KYSE-450 cells overexpressing PLAU were seeded in the upper chamber. After coculture for $48 \mathrm{~h}$, proteins were extracted from CAFs. Alternatively, the serum-free medium was replaced, and cells were incubated for $24 \mathrm{~h}$. Then, CM of CAFs was collected for further experiments.

RNA extraction and quantitative real time PCR (RT-qPCR)

RNA extraction and RT-qPCR were performed as described in a previous study ${ }^{25}$. The $2^{-\Delta \Delta C t}$ method was used to quantify the relative RNA expression level, and $\beta$-Actin served as an endogenous reference. All primers and oligonucleotides used in this study are listed in Supplementary Table S3.

\section{Western blotting}

Western blotting was performed as previously described $^{25}$. The primary antibodies used in the study are listed in Supplementary Table S4.

\section{Luminex liquid suspension chip detection}

$\mathrm{CM}$ of CAFs with or without PLAU treatment were used in these studies. Each sample was assessed in duplicate. Luminex liquid suspension chip detection was performed by Wayen Biotechnologies (Shanghai, China).

\section{Enzyme-linked immunosorbent assay (ELISA)}

$\mathrm{CM}$ of CAFs subject to various treatments (stimulated by drugs or CM of tumor cells, or cocultured with tumor cells) was collected for measurement of IL8 protein (without dilution) levels using the Human IL8 ELISA kit (ELH-IL8-1, RayBio). In addition, PLAU protein was measured in CM of knockdown and overexpressing PLAU KYSE-30, KYSE-450, and KYSE-180 cells and CM of wild type (WT) KYSE-30 and KYSE-450 cells using the PLAU ELISA kit (ELH-uPA-1, RayBio). Each sample was assessed in duplicate wells. Concentrations were calculated according to the manufacturer's instructions.

\section{RNA sequencing}

Here, shPLAU-1, -vec KYSE30 and KYSE450 cells were subject to mRNA sequencing (mRNA-seq) using Illumina HiSeq 4000. NFs and CAFs treated with PBS or recombinant PLAU were also assessed. mRNA-seq was performed by Boao (China).

\section{Cell proliferation and colony formation}

CCK8 assays (KeyGEN, China) were performed followed by the manufacturer's instructions. In brief, cells (KYSE-30: 2000 cells/well; KYSE-180 and KYSE-450: 2500 cells/well) were seeded into 96-well plates, and the OD values were measured at $0,24,48$, and $72 \mathrm{~h}$. OD values of KYSE-450 cells cocultured with CAFs were measured at $48 \mathrm{~h}$. For the colony formation assay, 200 cells were seeded into 6-well plates and cultured for 10-14 days. The colonies were fixed and stained with $1 \%$ crystal violet.

\section{Boyden chamber transwell assays}

In brief, we used 24-well Boyden chambers (Corning, USA) for migration assays as previously described ${ }^{25}$. Transfected cells (KYSE30, $1 \times 10^{4}$ or KYSE450, $5 \times 10^{4}$ ) were plated into the upper chamber and cultured in serum-free RPMI 1640 medium with or without treatment (cells overexpressing PLAU with U0126 added in the upper chamber). WT KYSE-30 and KYSE-450 cells were treated with CM of CAFs with or without recombinant PLAU in the presence or absence IPR-803. We counted the numbers of cells that migrated in five different areas at 100 -fold magnification.

\section{Tumor xenograft experiment}

All the animal experiments were approved by the Institutional Animal Care and Use Committee of the Cancer Hospital, Chinese Academy of Medical Sciences, and Peking Union Medical College. As previously described $^{26}, 1 \times 10^{6}$ shPLAU-1, -vec KYSE-30 cells, and KYSE-180 cells overexpressing PLAU and vector KYSE180 cells were subcutaneously injected into the flanks of mice (female 6-week-old to 8-week-old nude mice, $n=7$ per group) to establish tumor xenografts. In another experiment, MRC- 5 was stimulated with $20 \mathrm{ng} / \mathrm{ml}$ TGF $\beta 1$ for 4-5 days before further animal experiment. Briefly, $1 \times 10^{6}$ WT KYSE- 450 cells alone or $5 \times 10^{5}$ WT KYSE450 cells mixed with $5 \times 10^{5}$ MRC- 5 (or recombinant uPA pretreated MRC-5) were resuspended in $0.2 \mathrm{ml}$ of PBS and then subcutaneously injected into the flanks of mice to establish tumor xenograft (female 6-week-old to 8 -week-old nude mice, $n=7$ per group). Subgroups of 
mice were administered IPR-803 (50 mg/ $\mathrm{kg}$ ) via the intragastric route once every three days when the tumor reached $5 \mathrm{~mm}$ in diameter. The tumor volume was calculated by the formula: $V=\left(L \times W^{2}\right) / 2$. Four weeks later, all mice were sacrificed, and the tumor was removed and weighed.

\section{Lung colonization assay}

In brief, $1 \times 10^{6}$ shPLAU-1, -vec KYSE-30, and overexpressing $P L A U$ and vector KYSE- 180 cells were injected into female NOD-SCID mice (4-week-old to 6-week-old, six per group) through the tail vein. The mice were sacrificed 7 weeks later. The lungs were excised and fixed with $4 \%$ polysorbate and subsequently embedded in paraffin for hematoxylin and eosin (H\&E) staining. The number of lung surface metastatic nodes was calculated by gross and microscopic examination.

\section{Statistical analysis}

Prism GraphPad version 6.0, SPSS, GSEA, R scripting, and Cistrome network were used. Correlations between IL8 and PLAU expression were analyzed using Pearson's correlation coefficient. A chi square test was performed to determine the relationship between clinicopathological variables and PLAU expression. Overall survival (OS) curves were analyzed using the Kaplan-Meier method and log-rank tests. Significant differences between different groups were analyzed using two-tailed $t$-test or nonparametric Wilcoxon Sign-rank test. Data were presented as the mean standard deviation (SD). Differences were considered significant at $P<0.05,{ }^{* * * *} P<0.0001$, ${ }^{* * *} P<0.001$, ${ }^{* *} P<0.01$, and ${ }^{*} P<0.05$ (ns, not significant).

\section{Results}

High PLAU expression could indicate a poor prognosis in ESCC tissue

By analyzing GSE53625 data, we found that PLAU RNA expression was increased in cancer tissue compared with adjacent tissue (Fig. 1A). Furthermore, at the protein level, PLAU upregulation was evident only in three out of five derived tissue patients tested (Fig. 1B). PLAU immunohistochemical staining was performed on the ESCC tissue microarray, including 55 tumor and 50 adjacent cancer tissue samples, and the result were consistent with that noted at the RNA level (Fig. 1C). In addition, high PLAU expression predicted poor prognosis at both the RNA and protein expression levels (Fig. 1D, E). All these findings indicate that PLAU can serve as a prognostic marker of ESCC.

\section{PLAU was critical for ESCC cell proliferation and tumor growth in vitro and in vivo}

Previous studies have shown that PLAU can promote tumor proliferation in many tumors, such as breast cancer, and bladder cancer ${ }^{22,27}$. To explore PLAU function in ESCC, we detected PLAU expression levels in seven ESCC cell lines (Fig. 2A, B). Then, we constructed stable PLAU knockdown and overexpression ESCC cells and verified PLAU expression using WB and RT-qPCR (Fig. 2C, D). Given that PLAU is a secreted protein, we simultaneously assessed the supernatant of PLAU knockdown and overexpressing cells, and the results were consistent with that noted for total protein expression (Fig. 2E). There was no correlation between PLAU mRNA and protein expression in a number of cell lines. Using CCK8 experiments, we found that the proliferation of knockdown PLAU cells was reduced compared with the control, whereas the proliferation of cells overexpressing PLAU was enhanced (Fig. 2F, G). In addition, cells with PLAU knockdown and overexpression were subject to clone formation assays, and PLAU promoted the ability of ESCC cells to form clones (Fig. $2 \mathrm{H}, \mathrm{I})$. In vivo, the knockdown PLAU group exhibited reduced tumor growth and weight than the control group (Fig. 2J, K), while the PLAU overexpression group exhibited increased tumor growth and weight compared with the control group (Fig. 2L, M). These results show that PLAU promotes the growth of ESCC.

\section{PLAU promoted ESCC migration in vitro and in vivo}

$P L A U$ promotes migration in a variety of tumors ${ }^{27-29}$. Using Boyden chamber transwell assays, we found that the migration ability of cells with PLAU knockdown was decreased compared with the control (Fig. 3A, B), whereas cells overexpressing PLAU exhibit the opposite results (Fig. 3C, D). In addition, in vivo experiments showed that the number of pulmonary nodules in the knockdown PLAU group was less than that in the control group, whereas the number of pulmonary nodules in the PLAU overexpression group increased (Fig. 3E, F).

\section{PLAU promoted ESCC cell proliferation and migration via activation of the MAPK/MEK/Erk/Slug/MMP9 pathway}

To further explore the molecular mechanism by which PLAU promotes ESCC proliferation and migration, we performed RNA sequencing of shPLAU-1 and sh-vec KYSE-30 and KYSE-450 cells. Based on enrichment analysis, we hypothesized that the MAPK signaling pathway may be responsible for PLAU-mediated ESCC progression (Fig. 4A). Then, we verified the role of the classic MAPK signaling pathway. Western blot was used to detect the total and phosphorylated protein levels of c-raf, MEK, and Erk1/2 in knockdown and overexpressing PLAU ESCC cells, respectively. The results revealed reduced phosphorylated c-raf, MEK, and Erk1/2 levels in knockdown $P L A U$ cells compared with control, whereas the expression of these phosphorylated proteins was increased in cells overexpressing PLAU (Fig. 4B). Slug and Snail are common transcription factors that regulate invasion and 

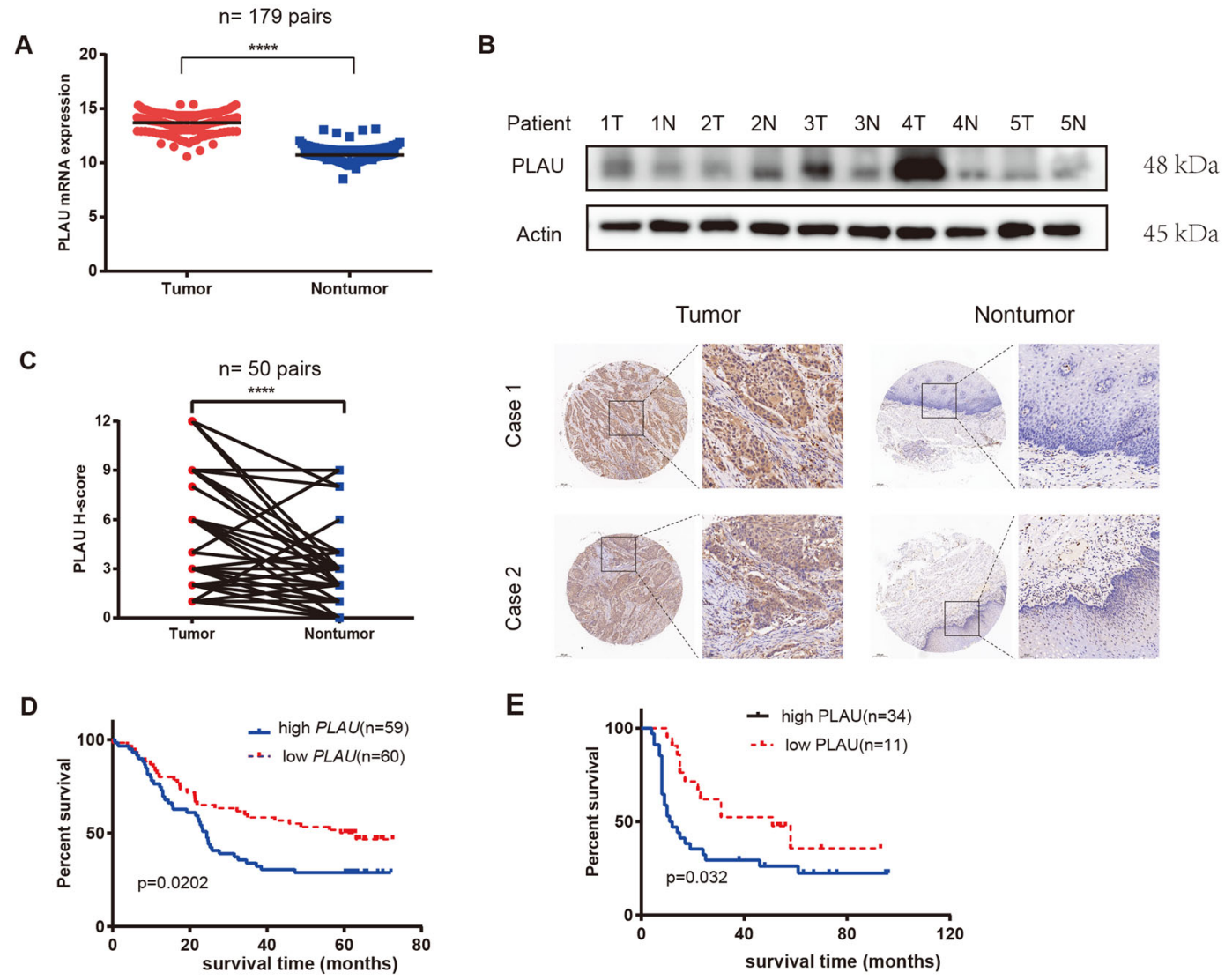

Fig. 1 High PLAU expression indicates a poor prognosis in ESCC tissue. A, B PLAU was more highly expressed in cancer compared with paracancer tissues at the RNA (A, GSE53625) and protein (B, C) levels, B western blot analysis of tumor and adjacent nontumor tissues from five patients, and $\mathbf{C}$ immunohistochemistry (IHC) scores (left) and representative images (right) of PLAU staining in ESCC tumor tissues and nontumor tissues (original magnification: 50x, 200x). D, E Kaplan-Meier survival analysis of overall survival based on high $(n=89)$ and low $(n=90)$ PLAU expression in GSE53625 (D) and high $(n=34)$ and low $(n=11)$ PLAU expression by IHC (E). ${ }^{*} P<0.05,{ }^{* *} P<0.01,{ }^{* * *} P<0.001,{ }^{* * *} P<0.0001$ (Student's $t$-test or).

metastasis in the downstream MAPK/MEK/Erk pathway. We further detected Slug and Snail expression levels in PLAU knockdown and overexpression cells. We found that PLAU upregulated Slug expression (Fig. 4B). MMPs is a common protein downstream of Slug that regulates migration. We found that MMP9 expression in PLAU knockdown cells was reduced compared with sh-vec cells, whereas MMP9 expression in cells overexpressing PLAU was increased (Fig. 4B). In addition, the highly selective MEK1/2 inhibitor U0126 reduce the high levels of MEK1/ 2 and Erk1/2 phosphorylation and Slug and MMP9 expression in cells overexpressing PLAU (Fig. 4C). In addition, U0126 also reversed $P L A U$-mediated promotion of ESCC cell proliferation, colony formation and migration (Fig. 4D-F).

\section{CAFs stimulated by PLAU promoted ESCC proliferation and migration}

PLAU in tumor cells further promotes ESCC cell proliferation and invasion by activating the classic MAPK pathway. Our previous studies demonstrate that the expression of PAI-1 and UPAR, both of which belong to the PLAU system, are significantly increased in CAFs and tumor cells, respectively, and promote ESCC cell proliferation and drug resistance ${ }^{8}$.

CAF heterogeneity is critical for promoting or inhibiting tumor, which is influenced by the paracrine system of other cells ${ }^{4,18,30,31}$. To further explore the effect of PLAU secreted by tumor cells on CAF heterogeneity, we detected the proliferation of ESCC cells cocultured with CAFs in the context of recombinant PLAU stimulation (Fig. 5A). CAFs subject to recombinant PLAU stimulation significantly promoted the proliferation of wild type (WT) KYSE-30 and KYSE-450 cells, and this effect was attenuated by the PLAU-uPAR inhibitor IPR-803 (Fig. 5B). After recombinant PLAU stimulation, conditional medium (CM) of CAFs also promoted migration of WT KYSE-30 and KYSE-450 cells, and IPR-803 eliminated this effect (Fig. 5C, D). Similarly, in vivo, subcutaneous tumor xenografts established from co-implantation of WT 


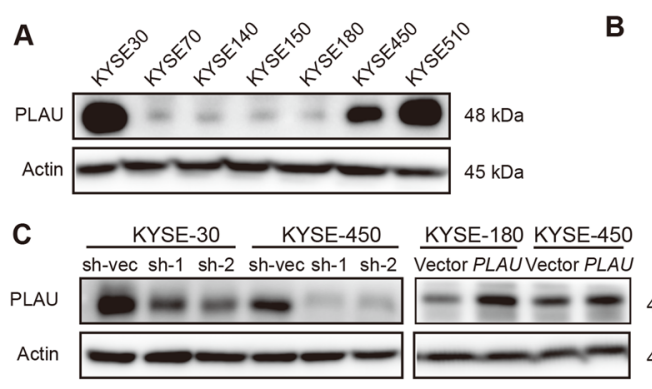

B

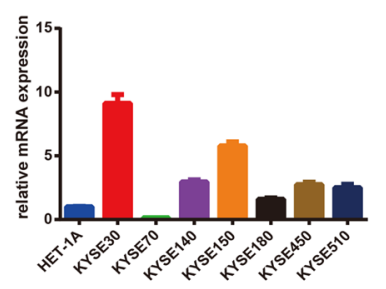

D
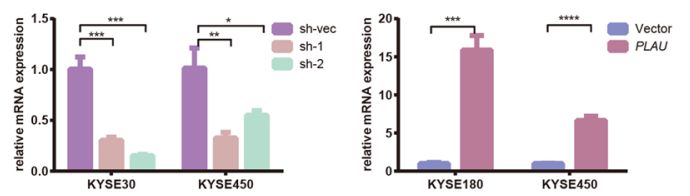

$48 \mathrm{kDa}$

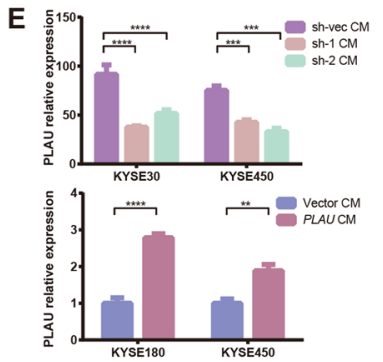

F

G

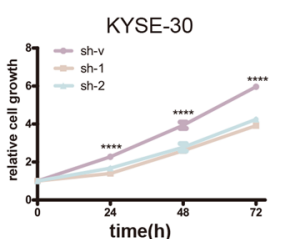

H
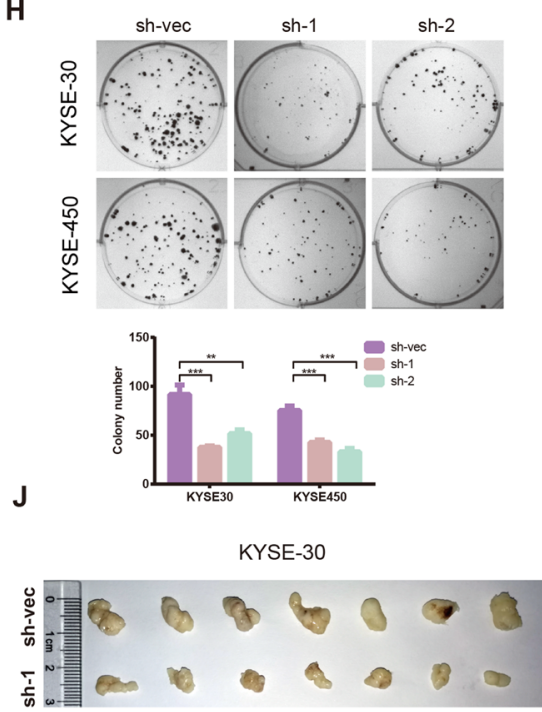

L

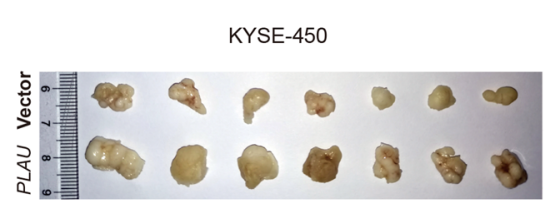

K

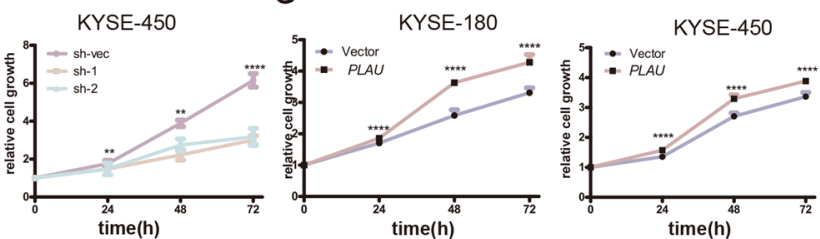

I
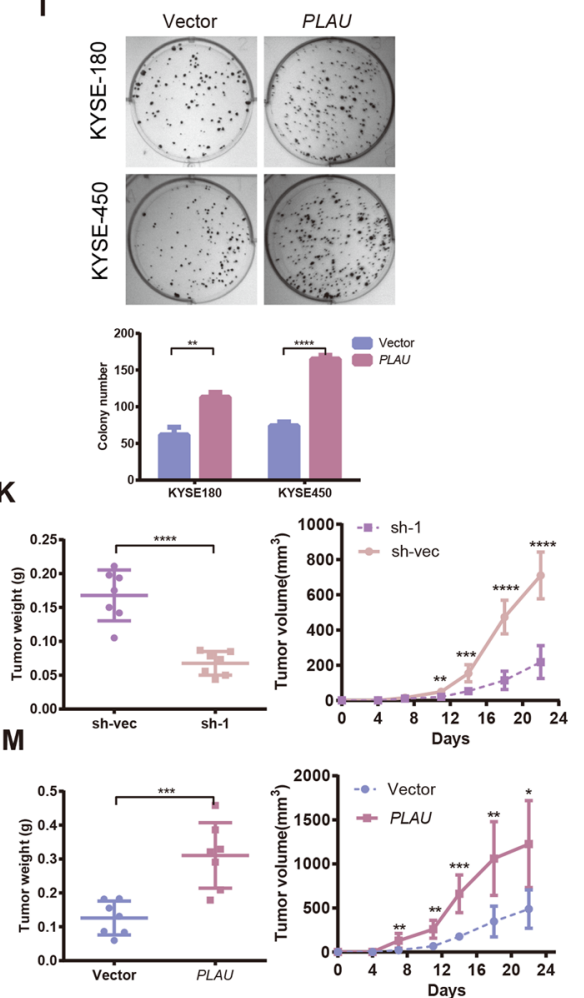

Fig. 2 PLAU was critical for ESCC cell proliferation and tumor growth. A, B Expression of PLAU in seven ESCC cell lines at the protein and RNA level (relative to HET-1A). C-E PLAU knockdown or overexpression in ESCC cells was verified by western blot (C), RT-qPCR (D), and ELISA (E). F, G Rate of cell growth of shPLAU-1, shPLAU-2 and sh-vec KYSE-30 and KYSE-450 cells and PLAU overexpressing and vector control cells were measured by CCK8 assays. H, I Colony formation of PLAU knockdown and overexpression KYSE-30, KYSE-450, and KYSE-180 cells. Colony numbers were compared between the groups. J-M Photos, weights, and growth curves of tumors in nude mice subcutaneously inoculated with shPLAU-1 and sh-vec KYSE-30 cells $(\mathbf{J}, \mathbf{K})$ or PLAU overexpressing or control cells (L, $\mathbf{M})$ (female 6-week-old to 8-week-old nude mice, $n=7$ per group). Three biological replicates were performed for in vitro assays. Data in bar charts are presented as the mean \pm SD. ${ }^{*} P<0.05,{ }^{*} P<0.01,{ }^{* * *} P<0.001$, ${ }^{* * *} P<0.0001$ (Student's $t$-test). 


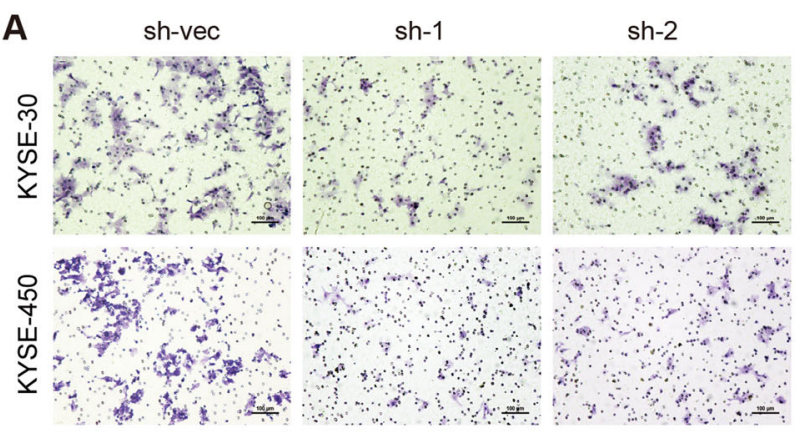

B

C

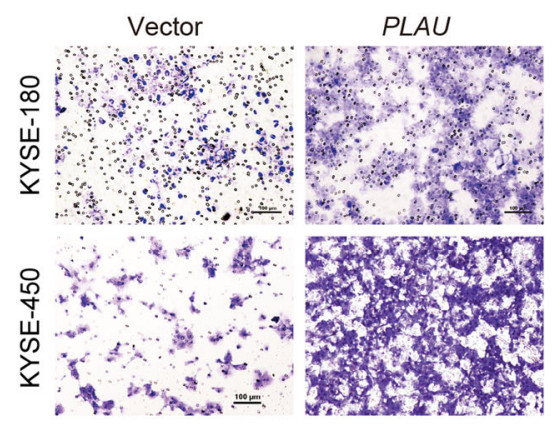

D

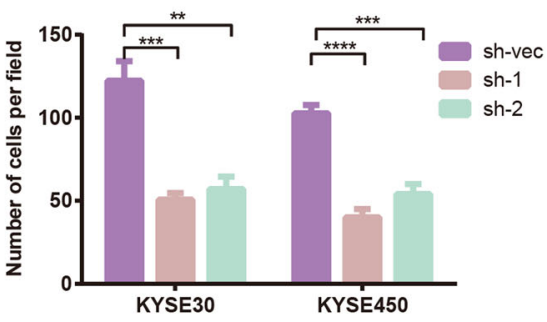

E
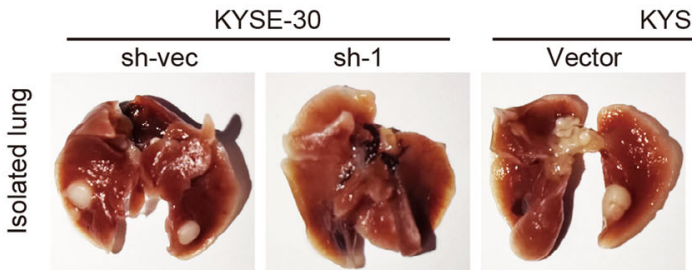

KYSE-180
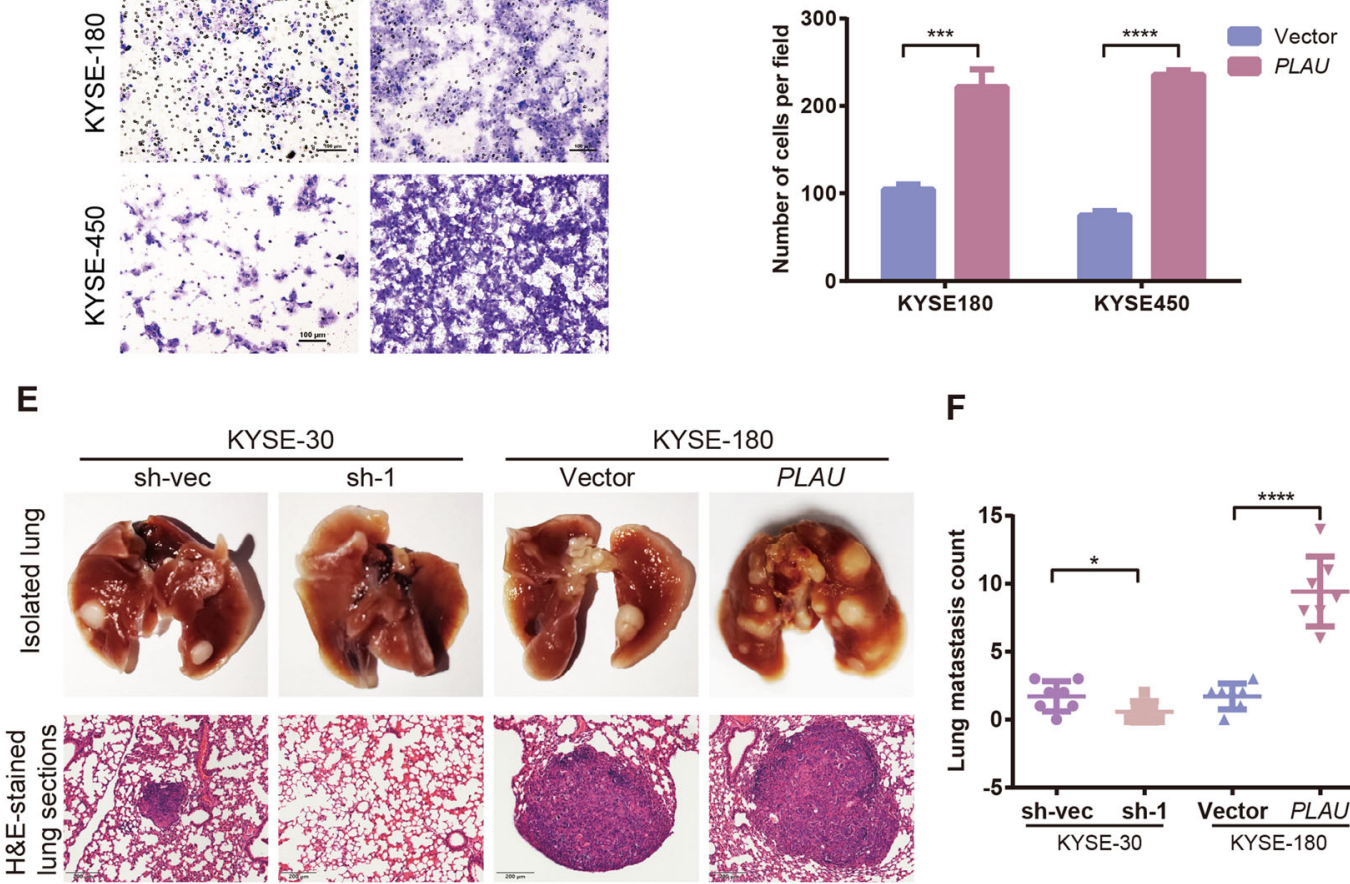

$\mathbf{F}$

Fig. 3 PLAU promoted ESCC cell migration in vitro and in vivo. A-D The migrating ability of shPLAU-1, shPLAU-2, sh-vec KYSE-30 and KYSE-450 cells (A) and PLAU overexpressing KYSE-180 and KYSE-450 cells (C) was detected using transwell chamber assays. The numbers of migrating cells were compared between the groups (B, D). E, F Representative images of lung tissues isolated from mice injected with $1 \times 10^{6}$ shPLAU-1 or sh-vec KYSE-30 cells, and PLAU overexpressing or vector KYSE-180 cells via the tail vein. Hematoxylin (the top panel) and eosin-stained images (200X, scale bars, $200 \mu \mathrm{m}$ ) of such tissues, and quantification of lung metastasis (female 4-week-old to 6-week-old NOID/SCID mice, $n=7$ per group). Three biological replicates were performed for in vitro assays. Data in bar charts are presented as the mean \pm SD. ${ }^{*} P<0.05,{ }^{* *} P<0.01,{ }^{* * *} P<0.001,{ }^{* * * *} P<$ 0.0001 (Student's t-test).

KYSE-450 and MRC-5 cells pretreated with TGF $\beta 1$ and PLAU grew faster than the controls without PLAU pretreatment, and this effect was also inhibited by IPR-803 (Fig. 5E-H).

\section{Tumor-secreted PLAU promoted the formation of inflammatory CAFs, which secreted IL8 via phosphorylation of Akt/NF-KB}

CAFs can be divided into inflammatory CAFs (iCAFs) with secretion phenotype and myofibroblasts (myCAFs) with contractility and matrix remodeling features ${ }^{17,18}$. To further explore the mechanism by which PLAU secreted by tumor cells promotes the tumor-promoting effect of CAFs, we performed RNA-seq of CAFs with or without recombinant uPA treatment and normal fibroblasts (NFs) obtained from the outgrowth of para-carcinoma tissues. CAFs interacts with tumor cells, and could promote progression of ESCC than NFs. iCAFs is promptingtumor subtype, whereas myCAFs is inhibiting-tumor subtype $^{17}$. Our results showed that compared with NFs, some gene sets of iCAFs, including of cytokines, chemokines, and interleukins, are highly expressed in CAFs, 
A

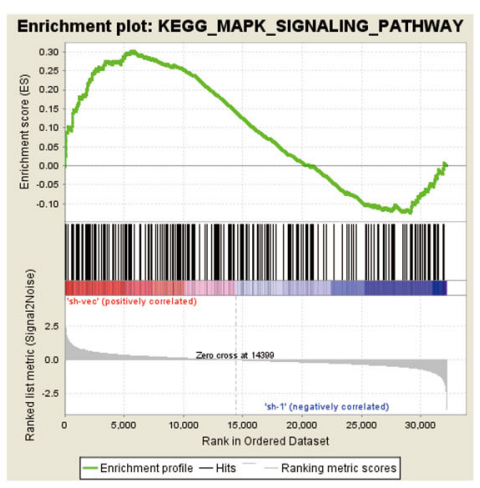

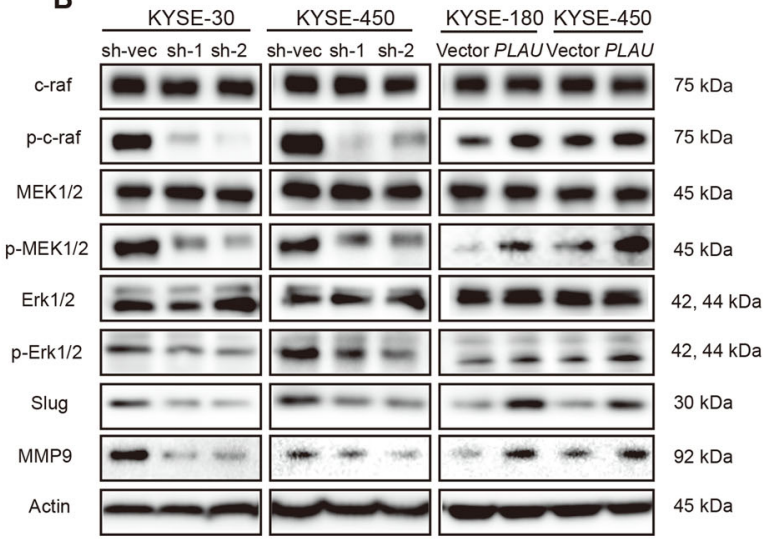

C

D

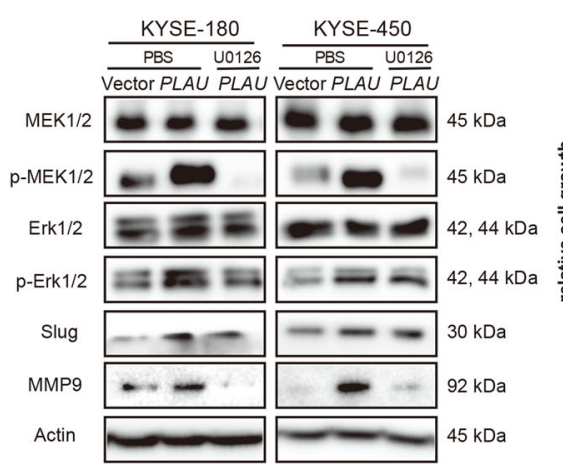

E
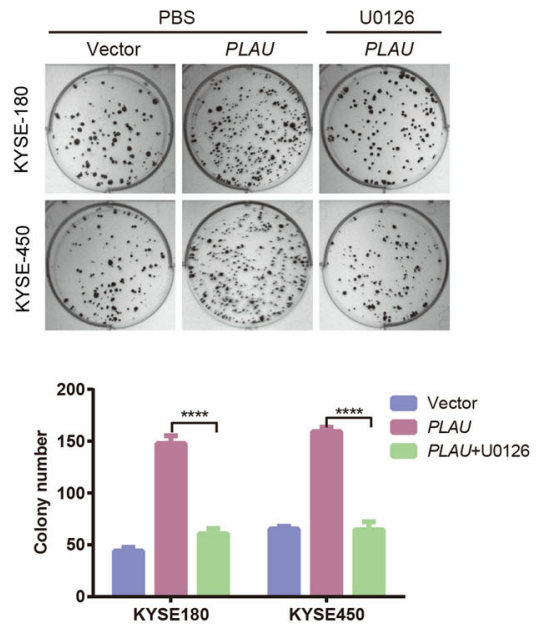
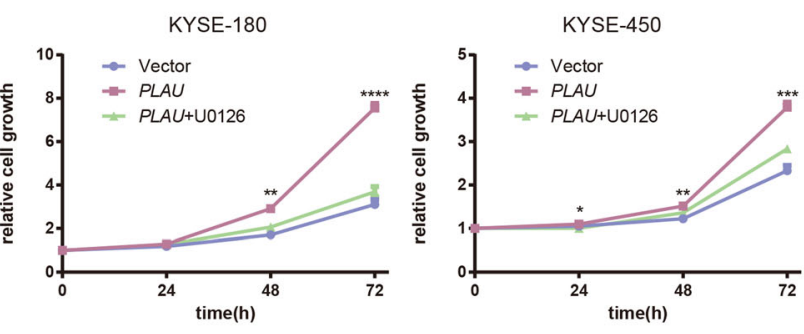

$\mathbf{F}$
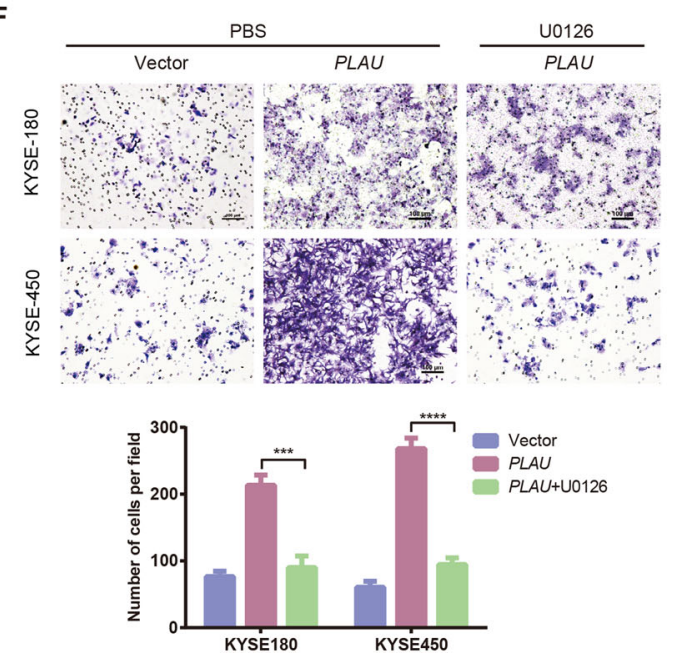

Fig. 4 PLAU promoted ESCC cell proliferation and migration via activation of the MAPK/MEK/Erk/Slug/MMP9 pathway. A MAPK signaling pathways enriched by sh-vec using GSEA. B, C Total and phosphorylated c-raf, MEK1/2, Erk1/2, Slug, and MMP9 expression levels in shPLAU-1, shPLAU2, and sh-vec KYSE-30 and KYSE-450 cells and overexpressing LAMC1 or vector KYSE-180 and KYSE-450 with (B) or without (C) U0126 treatment, a highly selective inhibitor of MEK1/2. D-F Proliferation, colony formation and migration of KYSE-180 and KYSE-450 cells overexpressing PLAU and vector with or without $\mathrm{U} 0126$ treatment. The number of colonies or migrating cells were compared between the groups (the bottom panel). Three biological replicates were performed for in vitro assays. Data in bar charts are presented as the mean \pm SD. ${ }^{*} P<0.05,{ }^{* *} P<0.01,{ }^{* * *} P<0.001,{ }^{* * * *} P<$ 0.0001 (Student's t-test). 
A

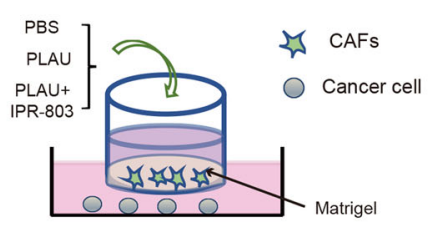

C

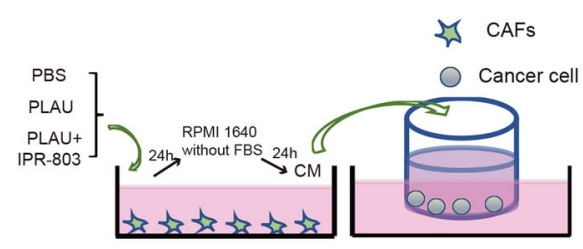

E

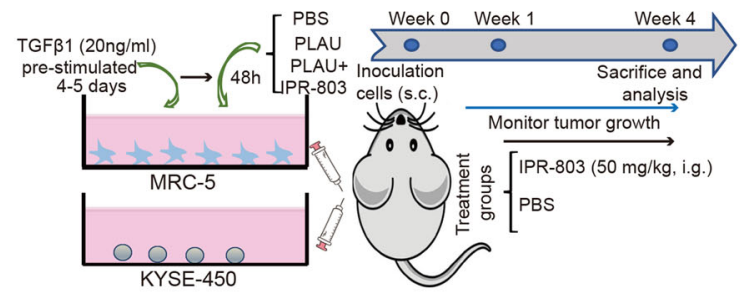

B

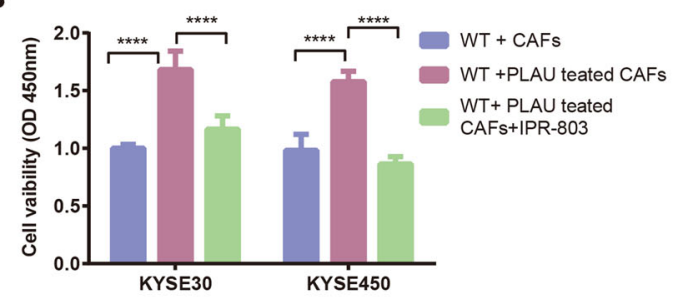

D

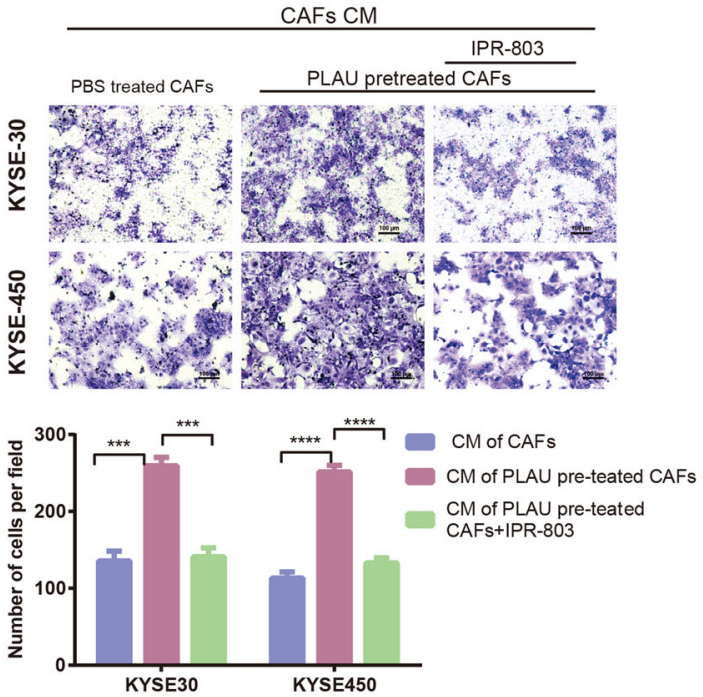

F

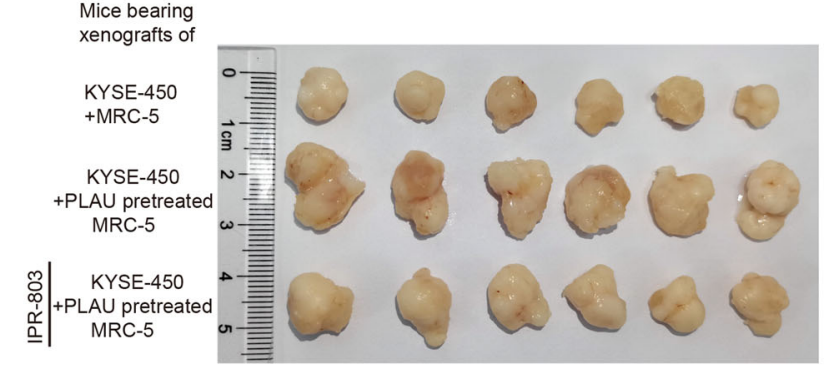

G

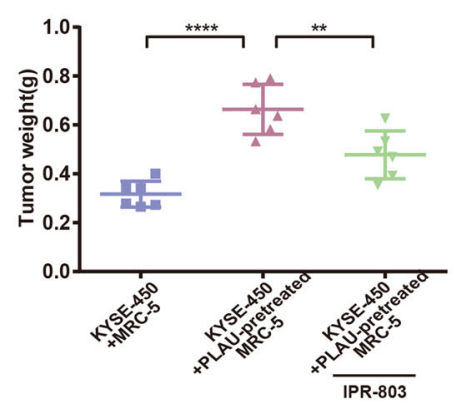

H

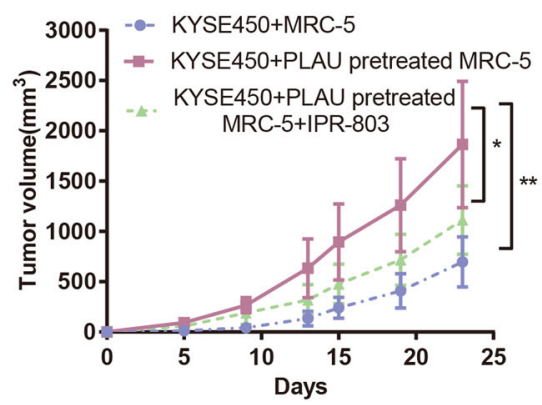

Fig. 5 CAFs stimulated by PLAU promoted ESCC proliferation and migration. A, B Experimental scheme and growth of WT KYSE-30 and KYSE450 cells cocultured with CAFs with or without recombinant PLAU in the presence or absence of the PLAU-uPAR inhibitor IPR-803 treatment. CCK8 assays were performed after co-culture for 48 h. C, D Experimental scheme and migration ability of WT KYSE-30 and KYSE-450 cells with CM of CAFs with or without recombinant PLAU pretreatment in the presence or absence of IPR-803. The numbers of migrating cells were compared between the groups (the bottom panel). E-H Outline experimental scheme (E), tumor volume (F), and weights $(\mathbf{G})$ and growth curves $(\mathrm{H})$ of subcutaneous tumor xenografts established from co-implantation of WT KYSE-450 cells, and indicated MRC-5 cells in the presence or absence of IPR-803 treatment (female 6-week-old to 8-week-old nude mice, $n=6$ per group). Three biological replicates were performed for in vitro assays. Three biological replicates were performed for in vitro assays. Data in bar charts are presented as the mean $\pm \mathrm{SD} .{ }^{*} P<0.05,{ }^{* *} P<0.01,{ }^{* * *} P<0.001,{ }^{* * *} P<0.0001$ (Student's $t$-test). 


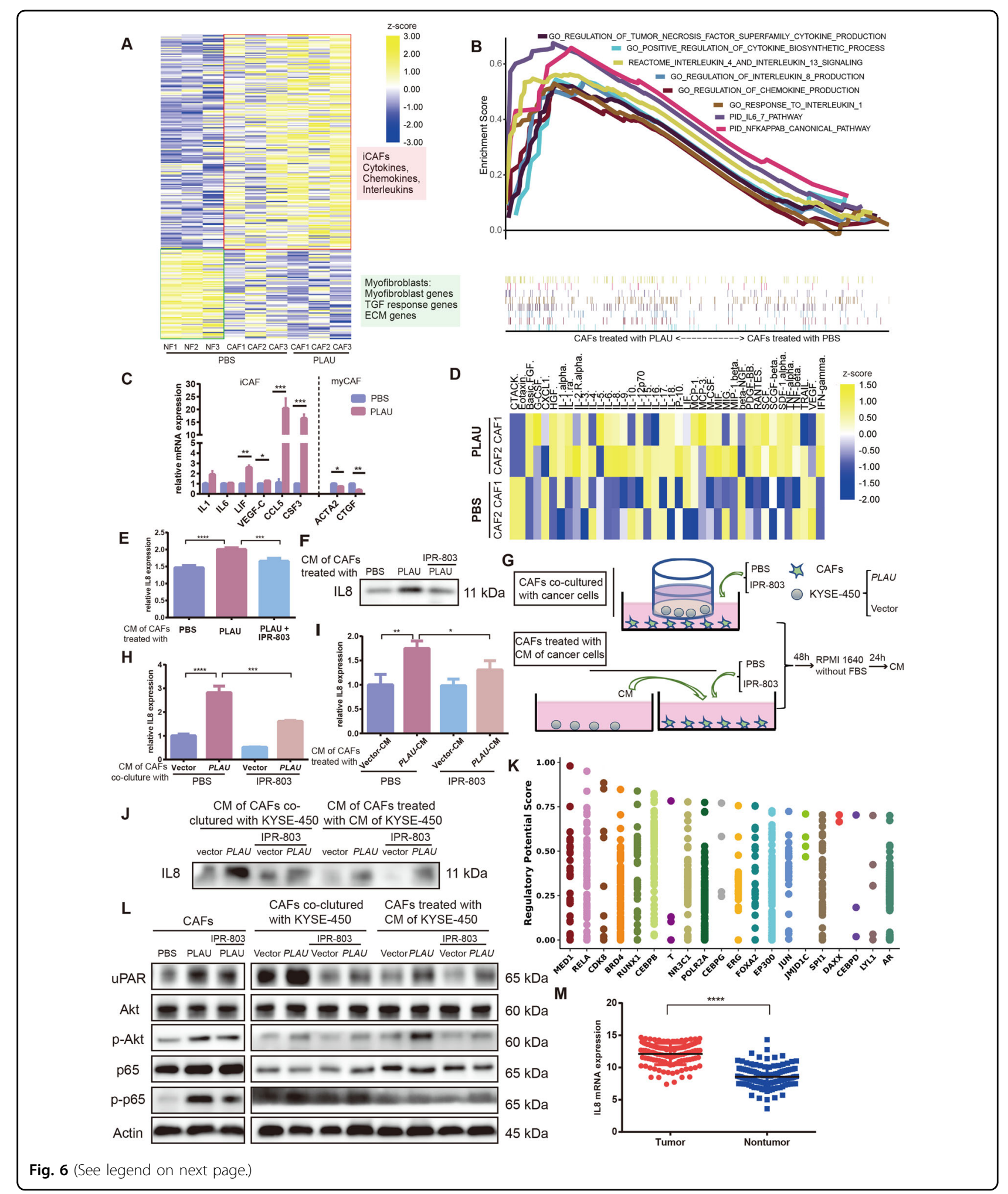

whereas some gene sets of myCAFs, including of myofibroblast genes, TGF $\beta$-responsive genes, and extracellular matrix (ECM) genes, exhibit the opposite effects. Furthermore, after recombinant PLAU stimulation, CAFs exhibit upregulation of gene sets of iCAFs and downregulation of gene sets of myCAFs (Fig. 6A). In addition, PLAU stimulated the activation of cytokine or chemokine pathways in CAFs (Fig. 6B). We hypothesized that PLAU 
(see figure on previous page)

Fig. 6 Tumor-secreted PLAU promoted the formation of inflammatory CAFs, which secreted IL8 via phosphorylation of Akt/NF-KB.

A Transcriptional profile of NFs and CAFs with or without recombinant PLAU treatment. B Recombinant PLAU promoted activation of cytokine, chemokine, and interleukins pathways and NF-KB canonical pathways as demonstrated by GSEA. C RT-qPCR detection of markers of iCAFs and myCAFs in CAFs with or without recombinant PLAU treatment. D A 48-cytokine panel was used to characterize the CM of CAFs with or without recombinant PLAU treatment. E, F Detection of IL8 expression in CM of CAFs with or without recombinant PLAU treatment in the presence or absence of IPR-803, a uPA-UPAR inhibitor, by ELISA (E) and western blot (F). G-J Experimental scheme (G) and detection of IL8 expression in CM of indicated CAFs cocultured with PLAU overexpressing and vector KYSE-450 cells $(\mathbf{H}, \mathbf{J})$ or treated with CM of PLAU overexpressing and vector KYSE-450 cells $(\mathbf{I}, \mathbf{J})$, in the presence or absence of IPR-803 as assessed by ELISA ( $\mathbf{H}-\mathbf{I})$ and western blot $(\mathbf{J})$. The CM used for western blots was concentrated 40fold. $\mathbf{K}$ Top 20 predicted transcription factors of CXCL1 predicted by the Cistome website. L Detection expression of uPAR, total and phosphorylated Akt and p65 of indicated CAFs. Three biological replicates were performed for in vitro assays. Data in bar charts are presented as the mean \pm SD. ${ }^{*} P<0.05,{ }^{* *} P<0.01,{ }^{* * *} P<0.001,{ }^{* * *} P<0.0001$ (Student's $t$-test).

induce secretion of cytokines and chemokines from CAFs. PLAU promotes the formation of iCAFs. We detected the expression of $\mathrm{iCAF}$ and myCAF markers in CAFs with or without recombinant PLAU stimulation by RT-qPCR. The results obtained were consistent with the sequencing results, but PLAU treated CAFs lacked of IL6 RNA upregulation (Fig. 6C). Additionally, we collected the CM of CAFs with or without recombinant PLAU treatment to assess the expression of 48 cytokines or chemokines. The results showed that compared with the control group, $\mathrm{CM}$ of CAFs with PLAU stimulation exhibited increased expression of various cytokines, of which IL8 exhibit the most obvious expression (Fig. 6D).

Furthermore, western blot and ELISA were performed to further confirm that PLAU promotes CAF-mediated secretion of IL8, which can be reversed by IPR-803, a specific inhibitor of PLAU-uPAR (Fig. 6E, F). By assessing IL8 expression in $\mathrm{CM}$ of CAFs cocultured with tumor cells overexpressing PLAU and in CAFs stimulated by $\mathrm{CM}$ of tumor cells overexpressing PLAU, we found that PLAU secreted by tumor cells promoted CAFs secretion of IL8 and that PLAU-uPAR specific inhibitor IPR-803 reversed the promotion effect (Fig. 6G-J). In addition, enrichment analysis showed that the NF- $\mathrm{kB}$ pathway may be activated in CAFs with PLAU treatment (Fig. 6B), and NF-kB could transcriptionally regulate IL8 as predicted on the Cistrome website (Fig. 6K). We detected the expression of uPAR and phosphorylated Akt and p65 in CAFs stimulated by recombinant PLAU, CAFs cocultured with tumor cells overexpressing $P L A U$, and CAFs treated with CM of tumor cells overexpressing PLAU. The results showed that PLAU upregulates the expression of uPAR and phosphorylated Akt and $\mathrm{p} 65$ in CAFs, which can be reduced by IPR-803 (Fig. 6L). In addition, IL8 expression was increased in ESCC cancer tissues than in paracancer tissues (Fig. 6M), but expression levels were not related to the patient's overall survival.

\section{IL8 secreted by CAFs upregulated PLAU expression in tumor cells}

Numerous previous studies demonstrated that IL8 promotes ESCC proliferation and invasion ${ }^{32-34}$. We had confirmed that PLAU secreted by tumor cells promotes IL8 secretion by CAFs. We further explored and found that PLAU expression was increased in WT KYSE-30 and KYSE-450 cells cocultured with CAFs with uPA treatment. The PLAU-uPAR inhibitor IPR-803 attenuated this effect (Fig. 7A, B). In addition, we found that recombinant IL8 upregulates PLAU expression in WT ESCC cells in a time-dependent and concentration-dependent manner (Fig. 7C-F). Increased PLAU expression mediated by IL8 is reversed upon treatment with SB225005, a CXCR2 inhibitor (Fig. 7G, H). IL8 also upregulated PLAU secretion of tumor cells, which is also reversed by SB225005, a CXCR2 inhibitor (Fig. 7I). By analyzing GSE53625 data, we found that IL8 expression in ESCC tissue was positively correlated with PLAU expression (Fig. 7J).

\section{Discussion}

In summary, PLAU promotes tumor cells proliferation and migration via MAPK/MEK/Erk/Slug/MMP9. Tumor cell secreted-PLAU promotes the conversion of fibroblasts to iCAFs that facilitate the expression and secretion of IL8 via uPAR/Akt/NF- $\mathrm{kB}$, and this activity can be can be blocked by a PLAU-uPAR inhibitor (IPR-803). IL8secreted by iCAFs upregulates PLAU expression (Fig. 8).

We found that overexpression of PLAU in ESCC is associated with poor prognosis, and PLAU potentially serves as a prognostic marker. Consistent with previous studies, high PLAU expression promotes the progression of $\mathrm{ESCC}^{35}$, and other various tumors, including breast, bladder, and lung cancer ${ }^{21,22,27}$. We found that PLAU promotes ESCC proliferation and tumor growth by activating the MAPK pathway. There is a close relationship between expression of PLAU and that of $\mathrm{p} 38 \mathrm{MAPK}^{36}$. High PLAU mRNA level is a characteristic of cancer cells with functional TRAIL signaling ${ }^{37}$. PLAU plays a role in promoting metastasis in various tumors ${ }^{27-29}$. PLAU expression is increased in breast cancer CTC cells ${ }^{28}$. We found that PLAU promotes migration in ESCC, and upregulates the expression of Slug and MMP9 via the MAPK pathway. MMP9 plays an important role in ECM remodeling, which could promote tumor metastasis ${ }^{38-40}$. 


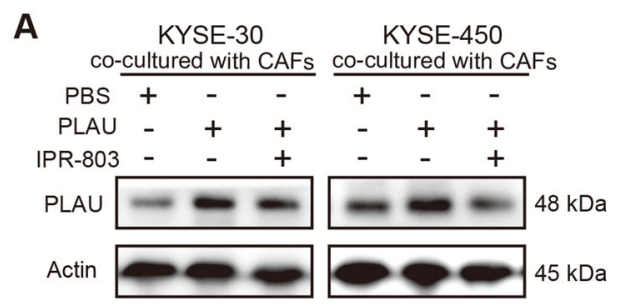

C

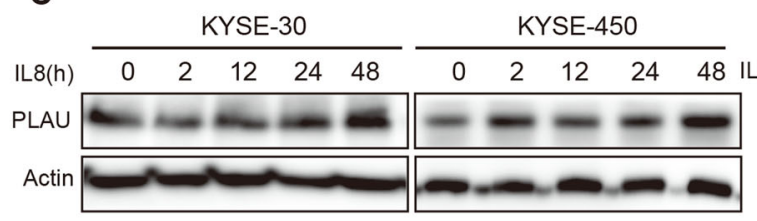

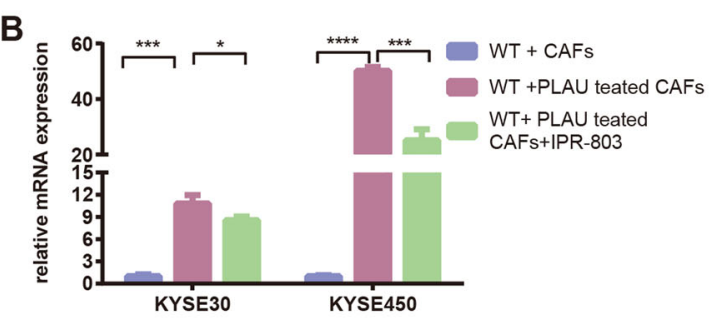

D
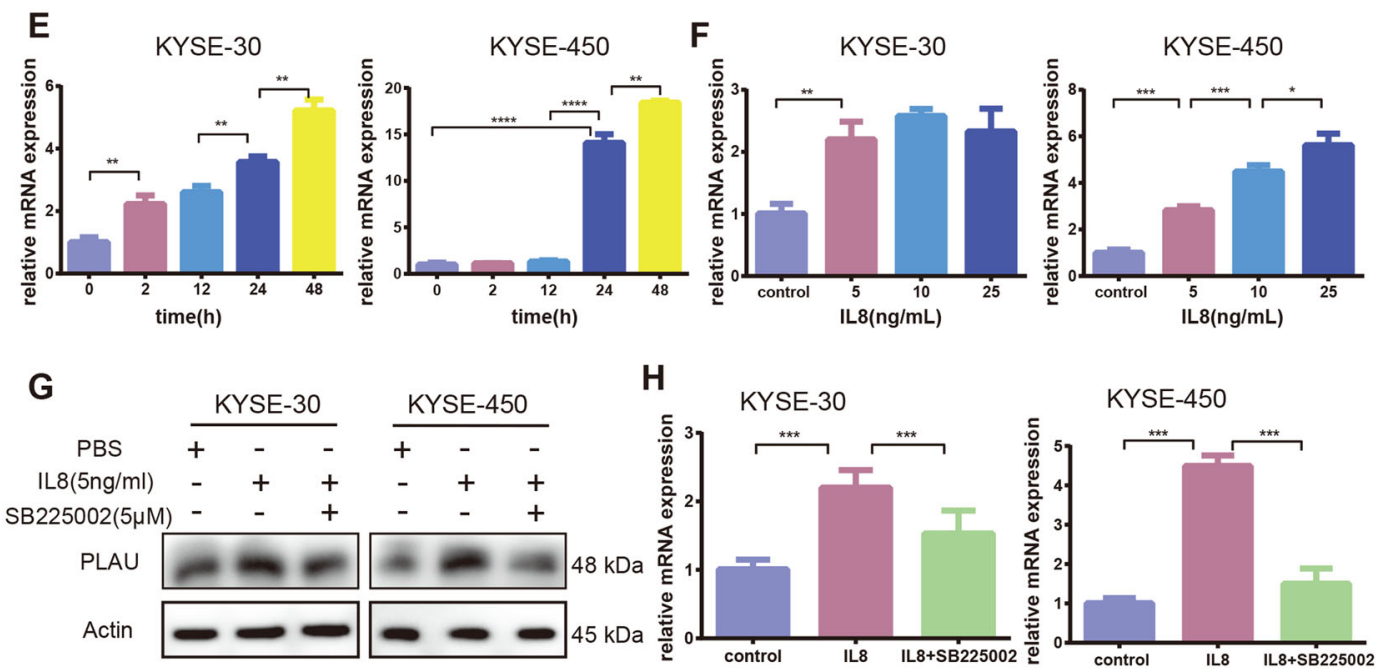

I
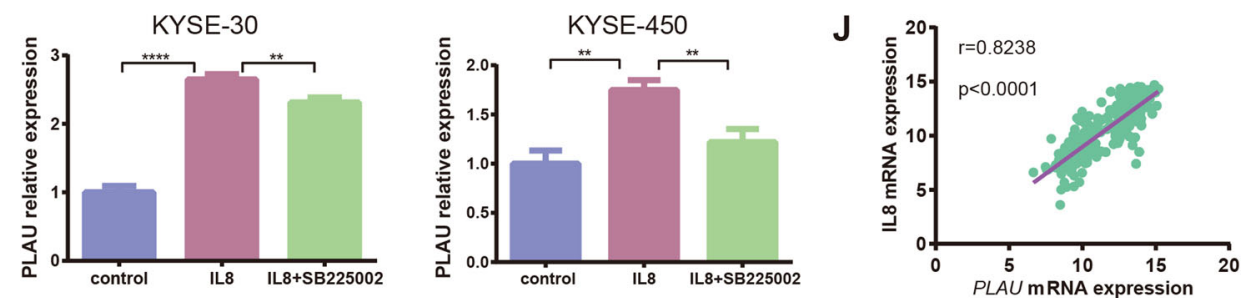

Fig. 7 IL8 secreted by CAFs upregulated PLAU expression in tumor cells. A, B Detection of PLAU expression of WT KYSE-30 and KYSE-450 cells cocultured with CAFs treated with recombinant UPA in the presence or absence of IPR-803, a uPA-UPAR inhibitor, as assessed by western blot (A) and RT-qPCR (B). C-F Western blot and RT-qPCR were performed to detect PLAU expression of WT KYSE-30 and KYSE-450 cells exposed to recombinant IL8 treatments at different times $(\mathbf{C}, \mathbf{E})$ and concentrations (D, F). G-I Detection of PLAU expression in WT KYSE-30 and KYSE-450 cells with recombinant IL8 treatment in presence or absence of SB225005, a CXCR2 inhibitor, by western blot (G), RT-qPCR (H), and ELISA (I). J Correlation analysis of IL8 and PLAU expression based on GSE53625 data. Three biological replicates were performed for in vitro assays. Data in bar charts are presented as the mean \pm SD. ${ }^{*} P<0.05,{ }^{* *} P<0.01$, ${ }^{* *} P<0.001,{ }^{* * * *} P<0.0001$ (Student's $t$ test).

Consistent with our results, previous studies have also confirmed that PLAU regulates ECM remodeling of the extracellular matrix, affects cell adhesion, and promotes tumor metastasis ${ }^{19}$. Additionally, previous studies have showed UPAR combined with integrins active many intercellular signals in tumor cells ${ }^{41}$, and c-Raf could be downstream of integrins ${ }^{42,43}$. The link between uPAR/ integrin and PLAU/c-Raf/MEK/Erk need furthermore research in the future.

CAFs are as heterogeneous as tumor cells ${ }^{44}$. Other cells influence CAF heterogeneity through paracrine methods. Different CAFs subgroups may secrete different cytokines to exert various functions, including promoting or inhibiting tumor progression ${ }^{16-18}$. In pancreatic cancers, 


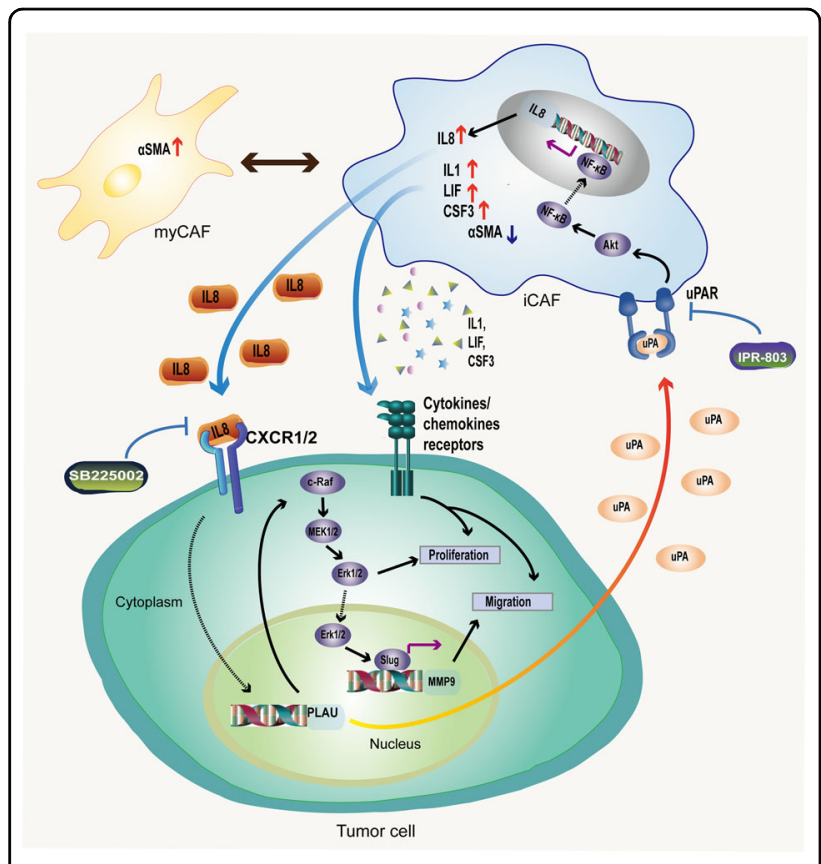

Fig. 8 Research summary: PLAU promotes tumor cells proliferation and migration via MAPK/MEK/Erk/Slug/MMP9.

Tumor cell secreted-PLAU promotes the conversion of fibroblasts to iCAFs that facilitate the expression and secretion of IL8 via UPAR/Akt/ NF-KB, and this activity can be can be blocked by a PLAU-UPAR inhibitor (IPR-803). IL8-secreted by iCAFs upregulates PLAU expression.

IL1 secreted by tumor cells promotes the conversion of fibroblasts around tumor cells into iCAFs by activating the JAK/STAT pathway ${ }^{18}$. We confirmed that PLAU secreted by tumor cells promotes the tumor-promoting effect of CAFs through the transformation of inflammatory CAFs. PLAU increases the expression of gene sets of iCAFs, but decreases the expression of gene sets of myCAFs. PLAU promotes the activation of cytokine, chemokine, and interleukin signaling pathways in CAFs. PLAU not only promotes the increase iCAF marker expression but also the reduces the expression of myCAF markers. Assessment of the CM of CAFs subject to PLAU treatment reveals increased expression of most cytokines and chemokines. All findings indicate that PLAU promotes the transformation of fibroblasts to iCAFs, which plays a tumor-promoting effect. Furthermore, we also found after PLAU treatment, two of NFs also have upregulation of gene sets of iCAFs and downregulation of myCAFs (Fig S1). PLAU promotes NF-kB pathway activation in CAFs, which is an upstream transcription factor that regulates a variety of cytokines and chemokines ${ }^{45}$. iCAF has secretory properties. It not only promotes tumor progression and deterioration through paracrine methods but may also be related to the systemic response of tumors ${ }^{18}$.
We also found that tumor secreted-PLAU promotes CAF-mediated secretion of IL8 via phosphorylation of NF- $\mathrm{KB}$. In addition, IL8 subsequently upregulates PLAU expression and secretion from tumor cells, which further promotes the progression of ESCC. IL8, which is also known as CXCL8, is a common chemokine that promotes neutrophil chemotaxis ${ }^{46}$. IL8 plays an important role in angiogenesis, survival, tumor stemness, invasion, metastasis, and immune cell infiltration in breast, lung, prostate, and pancreatic cancer ${ }^{46}$. In ESCC, IL8 directly binds to CXCR1/2 to promote invasion and migration $^{47,48}$. IL8 also recruits myeloid-derived suppressor cells by chemotaxis to promote tumor cell growth ${ }^{48}$. IL8 is associated with progression, metastasis, inflammation, and poor prognosis in ESCC patients ${ }^{47}$. CAFs stimulated by PLAU upregulates IL8 expression and secretion via uPAR/Akt/NF-kB. In esophageal carcinoma, IL8 and IL1 $\beta$ are the main downstream effectors of NF-kB ${ }^{40}$. In conclusion, PLAU could promote progression of tumor cell, and tumor cell secreted-PLAU promotes the conversion of fibroblasts to iCAFs, a promoting-tumor type of CAFs.

\section{Acknowledgements}

This work was supported by the Beijing Natural Science Foundation (7204291), the National Key Basic Research Development Plan (2018YFC1312105), the CAMS Innovation Fund for Medical Sciences (2017-12M-1-005, 2016-12M-1-001), the National Key R\&D Program of China (2016YFC1303201), the National Natural Science Foundation of China (81802299, 81502514, and 82002610), the Postgraduate Innovation Fund of Peking Union Medical College (2019-100254), the Fundamental Research Funds for the Central Universities (3332018070,3332020022).

\section{Conflict of interest}

The authors declare no conflict of interest.

\section{Publisher's note}

Springer Nature remains neutral with regard to jurisdictional claims in published maps and institutional affiliations.

Supplementary information The online version contains supplementary material available at https://doi.org/10.1038/s41420-021-00410-6.

Received: 16 November 2020 Revised: 18 December 2020 Accepted: 17 January 2021

Published online: 11 February 2021

\section{References}

1. Bray, F. et al. Global cancer statistics 2018: GLOBOCAN estimates of incidence and mortality worldwide for 36 cancers in 185 countries. Cancer J. Clin. 68, 394-424 (2018)

2. Chen, W. et al. Cancer statistics in China, 2015. Cancer J. Clin. 66, 115-132 (2016).

3. Lagergren, J., Smyth, E., Cunningham, D. \& Lagergren, P. Oesophageal cancer. Lancet 390, 2383-2396 (2017).

4. Kalluri, R. The biology and function of fibroblasts in cancer. Nat. Rev. Cancer $\mathbf{1 6}$, 582-598 (2016).

5. Nagarsheth, N., Wicha, M. S. \& Zou, W. Chemokines in the cancer microenvironment and their relevance in cancer immunotherapy. Nat. Rev. Immunol. 17, 559-572 (2017). 
6. Wang, W. et al. Effector T cells abrogate stroma-mediated chemoresistance in ovarian cancer. Cell 165, 1092-1105 (2016).

7. Zhang, $\mathrm{H}$. et al. CAF-secreted $\mathrm{CXCL} 1$ conferred radioresistance by regulating DNA damage response in a ROS-dependent manner in esophageal squamous cell carcinoma. Cell Death Dis. 8, e2790 (2017).

8. Che, Y. et al. Cisplatin-activated PAl-1 secretion in the cancer-associated fibroblasts with paracrine effects promoting esophageal squamous cell carcinoma progression and causing chemoresistance. Cell Death Dis. 9, 759 (2018).

9. Grugan, K. D. et al. Fibroblast-secreted hepatocyte growth factor plays a functional role in esophageal squamous cell carcinoma invasion. Proc. Natl Acad. Sci. USA 107, 11026-11031 (2010).

10. Qiao, Y. et al. IL6 derived from cancer-associated fibroblasts promotes chemoresistance via CXCR7 in esophageal squamous cell carcinoma. Oncogene 37, 873-883 (2018).

11. Shi, Y., Du, L., Lin, L. \& Wang, Y. Tumour-associated mesenchymal stem/stromal cells: emerging therapeutic targets. Nat. Rev. Drug Discov. 16, 35-52 (2017).

12. Ozdemir, B. C. et al. Depletion of carcinoma-associated fibroblasts and fibrosis induces immunosuppression and accelerates pancreas cancer with reduced survival. Cancer Cell 28, 831-833 (2015).

13. Paulsson, J. \& Micke, P. Prognostic relevance of cancer-associated fibroblasts in human cancer. Semin. Cancer Biol. 25, 61-68 (2014).

14. Chen, X. \& Song, E. Turning foes to friends: targeting cancer-associated fibroblasts. Nat. Rev. Drug Discov. 18, 99-115 (2019).

15. Amakye, D., Jagani, Z. \& Dorsch, M. Unraveling the therapeutic potential of the Hedgehog pathway in cancer. Nat. Med. 19, 1410-1422 (2013).

16. Kobayashi, H. et al. Cancer-associated fibroblasts in gastrointestinal cancer. Nat. Rev. Gastroenterol. Hepatol. 16, 282-295 (2019).

17. Ohlund, D. et al. Distinct populations of inflammatory fibroblasts and myofibroblasts in pancreatic cancer. J. Exp. Med. 214, 579-596 (2017).

18. Biffi, G. et al. IL1-induced JAK/STAT signaling is antagonized by TGFbeta to shape CAF heterogeneity in pancreatic ductal adenocarcinoma. Cancer Discov. 9, 282-301 (2019).

19. Mahmood, N., Mihalcioiu, C. \& Rabbani, S. A. Multifaceted role of the urokinase-type plasminogen activator (UPA) and its receptor (UPAR): diagnostic, prognostic, and therapeutic applications. Front. Oncol. 8, 24 (2018).

20. Sudol, M. From Rous sarcoma virus to plasminogen activator, src oncogene and cancer management. Oncogene 30, 3003-3010 (2011).

21. Wang, I. et al. Gene expression profiling in patients with chronic obstructive pulmonary disease and lung cancer. Am. J. Respir. Crit. Care Med. https://doi. org/10.1164/rccm.200703-3900C (2008)

22. Urban, P. et al. Increased expression of urokinase-type plasminogen activator mRNA determines adverse prognosis in ErbB2-positive primary breast cancer. J. Clin. Oncol. https://doi.org/10.1200/jco.2005.05.1912 (2006).

23. Sonnenblick, A. et al. Reactive stroma and trastuzumab resistance in HER2positive early breast cancer. Int. J. Cancer 147, 266-276 (2020).

24. Hannafon, B. N. et al. Exosome-mediated microRNA signaling from breast cancer cells is altered by the anti-angiogenesis agent docosahexaenoic acid (DHA). Mol. Cancer 14, 133 (2015).

25. Lu, Z. et al. The TGFbeta-induced InCRNA TBILA promotes non-small cell lung cancer progression in vitro and in vivo via cis-regulating HGAL and activating S100A7/JAB1 signaling. Cancer Lett. 432, 156-168 (2018).

26. Huang, J. et al. Interferon-inducible IncRNA IRF1-AS represses esophageal squamous cell carcinoma by promoting interferon response. Cancer Lett. 459, 86-99 (2019).
27. Chen, Z. et al. DANCR promotes metastasis and proliferation in bladder cancer cells by enhancing IL-11-STAT3 signaling and CCND1 expression. Mol. Ther. https://doi.org/10.1016/j.ymthe.2018.12.015 (2019).

28. Bredemeier, M. et al. Gene expression signatures in circulating tumor cells correlate with response to therapy in metastatic breast cancer. Clin. Chem. https://doi.org/10.1373/clinchem.2016.269605 (2017).

29. LeBeau, A. et al. Imaging active urokinase plasminogen activator in prostate cancer. Cancer Res. https://doi.org/10.1158/0008-5472.Can-14-2185 (2015).

30. Su, S. et al. CD10(+)GPR77(+) cancer-associated fibroblasts promote cancer formation and chemoresistance by sustaining cancer stemness. Cell $\mathbf{1 7 2}$ 841-856 (2018)

31. Kato, M. et al. Heterogeneous cancer-associated fibroblast population potentiates neuroendocrine differentiation and castrate resistance in a CD105dependent manner. Oncogene 38, 716-730 (2019).

32. Yung, M. M. et al. GRO-a and IL-8 enhance ovarian cancer metastatic potential via the CXCR2-mediated TAK1/NFKB signaling cascade. Theranostics 8 , 1270-1285 (2018)

33. Liu, Q. et al. The CXCL8-CXCR1/2 pathways in cancer. Cytokine Growth Factor Rev. 31, 61-71 (2016).

34. Ha, H., Debnath, B. \& Neamati, N. Role of the CXCL8-CXCR1/2 axis in cancer and inflammatory diseases. Theranostics 7, 1543-1588 (2017)

35. He, X., Xu, X., Zhu, G. \& Ye, H. Circulating uPA as a potential prognostic biomarker for resectable esophageal squamous cell carcinoma. Medicine $\mathbf{9 8}$ e14717 (2019).

36. Liu, Q., Li, W., Yang, S. \& Liu, Z. High expression of uPA related to p38MAPK in esophageal cancer indicates poor prognosis. OncoTargets Ther. 11, 8427-8434 (2018).

37. Pavet, V. et al. Plasminogen activator urokinase expression reveals TRAlL responsiveness and supports fractional survival of cancer cells. Cell Death Dis. https://doi.org/10.1038/cddis.2014.5 (2014).

38. Eble, J. A. \& Niland, S. The extracellular matrix in tumor progression and metastasis. Clin. Exp. Metastasis 36, 171-198 (2019).

39. Bonnans, C., Chou, J. \& Werb, Z. Remodelling the extracellular matrix in development and disease. Nat. Rev. Mol. Cell Biol. 15, 786-801 (2014).

40. Lin, E. W Karakasheva, T. A Hicks, P. D. Bass, A J. \& Rustgi, A. K. The tumor microenvironment in esophageal cancer. Oncogene 35, 5337-5349 (2016).

41. Smith, H. W. \& Marshall, C. J. Regulation of cell signalling by uPAR. Nat. Rev. Mol. Cell Biol. 11, 23-36 (2010).

42. Dastpeyman, $\mathbf{M}$. et al. Inhibition of silibinin on migration and adhesion capacity of human highly metastatic breast cancer cell line, MDA-MB-231, by evaluation of beta1-integrin and downstream molecules, Cdc42, Raf-1 and D4GDI. Med. Oncol. 29, 2512-2518 (2012).

43. Saher, G. \& Hildt, E. Activation of c-Raf-1 kinase signal transduction pathway in alpha(7) integrin-deficient mice. J. Biol. Chem. 274, 27651-27657 (1999).

44. Meacham, C. E. \& Morrison, S. J. Tumour heterogeneity and cancer cell plasticity. Nature 501, 328-337 (2013).

45. Taniguchi, K. \& Karin, M. NF-kappaB, inflammation, immunity and cancer: coming of age. Nat. Rev. Immunol. 18, 309-324 (2018).

46. Waugh, D. J. \& Wilson, C. The interleukin-8 pathway in cancer. Clin. Cancer Res. 14, 6735-6741 (2008)

47. Ogura, M. et al. Clinical significance of CXCL-8/CXCR-2 network in esophageal squamous cell carcinoma. Surgery 154, 512-520 (2013).

48. Li, P. et al. Maelstrom directs myeloid-derived suppressor cells to promote esophageal squamous cell carcinoma progression via activation of the Akt1/ RelA/L8 signaling pathway. Cancer Immunol. Res. 6, 1246-1259 (2018). 